\section{Original} article

\author{
Centre MURAZ, \\ Organisation de \\ Coordination et de \\ Coopération pour la \\ lutte contre les \\ Grandes Endémies \\ (OCCGE), \\ Bobo-Dioulasso, \\ Burkina Faso \\ I Sombié \\ N Meda \\ M Cartoux \\ $S$ Tiendrébéogo \\ A Ouangré \\ $S$ Yaro \\ O Ky-Zerbo \\ $P$ Van de Perre \\ Department of \\ Gynaecology and \\ Obstetrics, Centre \\ Hospitalier National \\ Sourô Sanou, \\ Bobo-Dioulasso, \\ Burkina Faso \\ B Dao
}

Maternité Port Royal, Groupe Hospitalier

Universitaire Cochin

Port-Royal, Paris,

France

L Mandelbrot

Unité INSERM 330,

Université Victor

Segalen Bordeaux2,

Bordeaux, France

F Dabis

${ }^{\star}$ Other members listed at end of paper.

Correspondence to: Nicolas Meda, Centre MURAZ/OCCGE, $01 \mathrm{PO}$

Box 153 Bobo-Dioulasso 01, Burkina Faso, West Africa nmeda.muraz@fasonet.bf

Accepted for publication 25 February 2000

\title{
Seroprevalence of syphilis among women attending urban antenatal clinics in Burkina Faso, 1995-8
}

\author{
I Sombié, N Meda, M Cartoux, S Tiendrébéogo, A Ouangré, S Yaro, O Ky-Zerbo, B Dao,
} $P$ Van de Perre, L Mandelbrot, F Dabis, for the DITRAME Study Group ${ }^{\star}$

Objectives: To describe annual trends in syphilis seroprevalence and to identify risk factors of syphilis among pregnant women receiving antenatal care in Bobo-Dioulasso, Burkina Faso.

Methods: Women were recruited between January 1995 and July 1998 in three antenatal clinics where counselling and HIV testing services had been established in the context of a trial evaluating a short course of zidovudine to reduce mother to child transmission of HIV (ANRS 049 trial). Sociodemographic variables were collected during HIV pretest counselling sessions. Syphilis diagnosis was considered when serum was positive with both rapid plasma reagin and Treponema pallidum haemagglutination assay (TPHA) tests.

Results: Overall, 10980 pregnant women were screened. Syphilis seroprevalence was $0.24 \%$ (95\% confidence interval (CI): $0.15-0.35$ ) without changes over time. HIV prevalence was $8.8 \%$ (CI: 8.3-9.3). In a multivariable analysis, having casual sex partners (odds ratio $(\mathrm{OR})=4.48$; CI: 1.62-12.38), being HIV seropositive ( $\mathrm{OR}=2.62$; CI: $1.02-6.74)$, and being illiterate $(\mathrm{OR}=3.78$; CI: 1.24-11.48) were independent risk factors for syphilis infection.

Conclusions: This study suggests low syphilis seroprevalence in this city of Burkina Faso. Sexually transmitted disease programmes should be reinforced to offer free access to syphilis screening and treatment in order to eliminate this disease, in coordination with HIV prevention and care. (Sex Transm Inf 2000;76:314-316)

Keywords: syphilis; HIV; pregnant women; Burkina Faso

\section{Introduction}

Syphilis remains a serious public health problem in sub-Saharan Africa. ${ }^{1}$ Epidemiological data show clearly an area of high prevalence in eastern and southern Africa and another one of low prevalence in western and northern Africa. ${ }^{2}$ Goeman et al note that demographic, sociological, economic, political, biological, medical, behavioural, and educational factors influence this ecological difference in syphilis epidemiology. ${ }^{2}$

As part of an HIV research programme, over 4 years we provided syphilis screening to all pregnant women attending prenatal clinics in Bobo-Dioulasso, Burkina Faso, west Africa. This work aimed at estimating the prevalence and annual trends of syphilis with a view to verifying the lowering prevalence suggested in the literature, and identifying factors associated with syphilis in pregnant women in this population.

\section{Methods}

Within the framework of a randomised placebo controlled trial evaluating a short regimen of zidovudine to reduce mother to child transmission of HIV (ANRS 049 trial), services for voluntary and confidential HIV testing were established in three mother and child health centres in Bobo-Dioulasso. Our method for enrolling and testing women for HIV infection has already been described. ${ }^{3}$ During pretest counselling sessions a standardised questionnaire was administered to collect sociodemographic and behavioural characteristics of pregnant women. Syphilis serology was performed using rapid plasma reagin (RPR) slide test and Treponema pallidum haemagglutination assay (TPHA) provided by BioMerieux (Paris, France). All subjects dually reactive to RPR slide test and TPHA were considered as having active syphilis.

Data were analysed using the software packages EPI-INFO and EGRET. Estimates of syphilis and HIV seroprevalence are presented with their $95 \%$ confidence interval (CI). The $\chi^{2}$ test for trend was used to compare yearly seroprevalence of syphilis from 1995 to 1998 . The Pearson $\chi^{2}$ test and two tailed Fisher's exact test were applied to compare women with and without syphilis in terms of sociodemographic and behavioural data. Logistic regression analysis was used to look for independent risk factors for syphilis infection and to adjust for potential confounders. Since stratified analysis did not shown any interaction, no interaction term was included in the multivariate model.

\section{Results}

Over the 4 years of the programme, HIV and syphilis screening was offered to 11854 women in total and 10990 (92.7\%) accepted the offer. Ten could not be tested because of broken test tubes, so our study involved 10980 women. Their average age was 25.7 years. The mean number of pregnancies was 3.5 and mean number of deliveries, 2.4. Fifteen per cent of the women had a history of abortion and $3.3 \%$ a history of stillbirth. Sixty five per cent of women were illiterate. Most women 
Table 1 Syphilis infection observed between 1995 and 1998 among pregnant women, Bobo-Dioulasso, Burkina Faso *

\begin{tabular}{llll}
\hline & No of women & Prevalence (\%) & $95 \%$ CI \\
\hline 1995 & 2147 & 0.42 & $0.19-0.79$ \\
1996 & 2486 & 0.28 & $0.11-0.58$ \\
1997 & 3946 & 0.13 & $0.04-0.29$ \\
1998 & 2396 & 0.21 & $0.07-0.48$ \\
Total & 10980 & 0.24 & $0.15-0.35$ \\
\hline
\end{tabular}

${ }^{\star} \chi^{2}$ for trend from 1995 to $1998: 3.347, \mathrm{p}$ value $=0.07$.

were married $(88.4 \%)$. Six per cent of pregnant women reported condom use in the past year and $8 \%$ reported sex with a casual partner in the past 12 months. Finally, 11\% of women had travelled outside Bobo-Dioulasso in the past year.

Only 26 women $(0.24 \%$, CI: $0.15-0.35)$ had syphilis. Table 1 shows annual syphilis infection seroprevalence observed between 1995 and 1998. Trend analysis showed no significant decrease in the syphilis infection rate $(\mathrm{p}=0.07)$. The seroprevalence of syphilis among pregnant women remained low and stable. In all, 975 women $(8.8 \%)$ had a positive HIV serology. Five women were infected both with HIV and syphilis.

RISK FACTORS FOR SYPHILIS

Table 2 presents results from single and multivariable logistic regression analyses that have compared women with positive and negative syphilis serology with regard to sociodemographic and behavioural characteristics. The two groups of women were similar for age, obstetric history, marital status, and whether they reported condom use or travel in the past year or not. After adjusting for other factors, having occasional sexual partners, being illiterate, and having HIV seropositive were independent risk factors for the presence of syphilis infection among pregnant women in BoboDioulasso.

\section{Discussion}

Our study, which was carried out from 1995 to 1998, confirms the low prevalence of syphilis infection among pregnant women in Burkina Faso. HIV infection, illiteracy, and having occasional sexual partners were the independent risk factors for syphilis in our study population.

Selection and assessment biases could cast doubts about the reality of the very low syphilis seroprevalence observed. Indeed, we have used voluntary confidential HIV tests and women who saw themselves as at risk may have excluded themselves or refused the test. However, acceptability was high (93\%) and even in the extreme scenario of the $7 \%$ who refused being syphilis positive, the seroprevalence of syphilis would still remain low by African standards. The diagnostic strategy is unlikely to have underestimated the seroprevalence of syphilis since the method used is universally accepted. ${ }^{4}$ Therefore, we believe that the seroprevalence of $0.24 \%$ that was found reflects the reality in Bobo-Dioulasso and confirms low seroprevalence of syphilis among pregnant women in Burkina Faso as previously reported. ${ }^{5}$ This is in agreement with the mapping of the prevalence of syphilis infection reported by Goeman et $a l^{2}$ and the findings of recent seroprevalence studies. ${ }^{67}$

Table 2 Factors associated with syphilis infection among pregnant women in Bobo-Dioulasso, Burkina Faso, 1995-8

\begin{tabular}{|c|c|c|c|c|c|c|}
\hline \multirow[b]{2}{*}{ Characteristic } & \multirow{2}{*}{$\begin{array}{l}\text { Syphilis } \\
\text { serology } \\
(n=26)\end{array}$} & \multirow{2}{*}{$\begin{array}{l}\text { Syphilis } \\
\text { serology (-) \% } \\
(n=10980)\end{array}$} & \multicolumn{2}{|c|}{ Single variable analysis } & \multicolumn{2}{|c|}{ Multivariable analysis } \\
\hline & & & OR $(95 \% C I)^{\star}$ & $p$ Value & OR $(95 \% C I)^{\star}$ & $p$ Value \\
\hline \multicolumn{7}{|l|}{ Age } \\
\hline$<20$ years & 19.2 & 16.5 & $1.1(0.4-3.6)$ & 0.82 & $1.2(0.3-5.9)$ & 0.82 \\
\hline $20-29$ years & 53.8 & 57.1 & $0.9(0.4-2.3)$ & 0.92 & $1.1(0.4-3.0)$ & 0.92 \\
\hline$\geqslant 30$ years & 26.9 & 26.4 & 1.0 & & 1.0 & \\
\hline \multicolumn{7}{|l|}{ Number of pregnancies } \\
\hline 1 & 23.1 & 22.4 & $1.0(0.4-2.6)$ & 0.97 & $1.1(0.1-10.5)$ & 0.95 \\
\hline 2 & 19.2 & 20.8 & $0.9(0.3-2.5)$ & 0.86 & $0.4(0.0-8.1)$ & 0.57 \\
\hline$\geqslant 3$ & 57.7 & 56.9 & 1.0 & & 1.0 & \\
\hline \multicolumn{7}{|l|}{ Number of deliveries } \\
\hline 0 & 30.8 & 24.3 & $1.3(0.5-3.1)$ & 0.56 & $2.9(0.2-44.6)$ & 0.45 \\
\hline 1 & 15.4 & 21.5 & $0.7(0.2-2.2)$ & 0.58 & $0.7(0.1-6.3)$ & 0.73 \\
\hline$\geqslant 2$ & 53.8 & 54.3 & 1.0 & & 1.0 & \\
\hline \multicolumn{7}{|l|}{ Number of miscarriages } \\
\hline 0 & 73.1 & 85.2 & 1.0 & & 1.0 & \\
\hline$\geqslant 1$ & 26.9 & 14.8 & $2.1(0.9-5.1)$ & 0.08 & $1.8(0.6-5.4)$ & 0.31 \\
\hline \multicolumn{7}{|l|}{ Number of stillbirths } \\
\hline 0 & 96.2 & 96.8 & 1.0 & & 1.0 & \\
\hline$\geqslant 1$ & 3.8 & 3.2 & $1.2(0.2-8.8)$ & 0.86 & $0.9(0.1-7.1)$ & 0.94 \\
\hline \multicolumn{7}{|l|}{ Marital status } \\
\hline Single/separated/widowed & 11.5 & 11.6 & 1.0 & & 1.0 & \\
\hline Married, living together & 88.5 & 88.4 & $1.0(0.3-3.4)$ & 0.98 & $1.0(0.3-3.6)$ & 0.98 \\
\hline \multicolumn{7}{|l|}{ Literacy } \\
\hline Illiterate & 84.5 & 65.2 & $2.9(1.0-8.5)$ & 0.04 & $3.8(1.2-11.5$ & 0.02 \\
\hline Literate & 15.4 & 34.8 & 1.0 & & 1.0 & \\
\hline \multicolumn{7}{|c|}{ Number of casual partners in the past year } \\
\hline 0 & 69.2 & 92.2 & 1.0 & & 1.0 & \\
\hline$\geqslant 1$ & 30.8 & 7.8 & $5.3(2.3-12.1)$ & $<0.001$ & $4.5(1.6 .12 .4)$ & $<0.01$ \\
\hline \multicolumn{7}{|l|}{ Condom use in the past year } \\
\hline Yes & 7.7 & 6.5 & 1.0 & & 1.0 & \\
\hline No & 92.3 & 93.5 & $0.8(0.2-5.1)$ & 0.68 & $0.5(0.1-2.5)$ & 0.43 \\
\hline \multicolumn{7}{|l|}{ Travel in the past year } \\
\hline Yes & 30.8 & 11.0 & $3.6(1.6-8.3)$ & $<0.01$ & $2.2(0.8-5.9)$ & 0.12 \\
\hline No & 69.2 & 89.0 & 1.0 & & 1.0 & \\
\hline \multicolumn{7}{|l|}{ HIV status } \\
\hline Seropositive & 23.1 & 8.8 & $3.1(1.3-7.8)$ & 0.01 & $2.6(1.0-6.7)$ & 0.04 \\
\hline Seronegative & 76.9 & 91.2 & 1.0 & & 1.0 & \\
\hline
\end{tabular}

^Odds ratio $(95 \% \mathrm{CI})$. 
The unrestricted sale of drugs in the market place is increasing in Africa and particularly in Burkina Faso. It induces the extensive use of antibiotics, especially of ampicillins which can stop syphilis and contribute towards reducing syphilis seroprevalence. ${ }^{2}$ In addition, the numerous campaigns promoting safe sex and condoms conducted since the onset of the HIV/AIDS epidemic have led to changes in attitudes and practices at the community level. ${ }^{8}$ In our study site, the sale of condoms increased from 532224 units in 1993 to 1682208 in 1998 , another possible contributing factor to the decreasing incidence of syphilis.

Strengthening the treatment strategy for syphilis control requires identifying related factors, which would help in the adoption of adequate and efficient measures. The only factors that were identified in our study with very low syphilis prevalence were HIV infection, having casual sexual partners, and illiteracy. Since information, education, and communication campaigns are most often performed in French through the media and posters in our population, the illiterates are the least informed. Changes in sexual behaviour are therefore quite slow in this subgroup, which exposes them to all sexually transmitted infections (STIs). The association found among syphilis, behavioural factors, and HIV infection has already been well documented. ${ }^{5} 910$

This low prevalence of syphilis infection among pregnant women indicates clearly that the elimination of syphilis is possible in this population if the following measures are taken: the reinforcement of maternal and child health services where serological screening of syphilis is done routinely; treatment of the positive female cases; screening of partners and treatment of the positive ones. The experience of Mwanza in Tanzania showed that it is possible with a comprehensive programme for STI syndromic management to decrease both STI prevalence and HIV incidence. ${ }^{11}{ }^{12}$ The widespread use of this efficient intervention in Burkina Faso could help greatly to eliminate syphilis and impact on other STIs.

In conclusion, our study confirms the low prevalence of syphilis among pregnant women in the second largest city of Burkina Faso. The factors associated with syphilis are accessible to information, education, and communication intervention programmes. Combined with serological screening of pregnant women and syndromic management of STIs, rapid elimination of syphilis and reduction of the incidence of HIV are reasonable targets.

\section{Appendix}

The DITRAME (DIminution de la TRAnsmission Mère-Enfant) Study Group is organised as follows: 1: Abidjan Centre, 2: Bobo-Dioulasso Centre, 3: Bordeaux Coordination Unit, 4: Paris.
Biostatistics: L Dequae-Merchadou, ${ }^{3}$ R Lassalle ${ }^{3}$, V Leroy ${ }^{3}$, R Salamon 3 .

Epidemiology: $\mathrm{M}$ Cartoux ${ }^{2}, \mathrm{~F}$ Dabis $^{3}$ (Coordinator of the DITRAME ANRS 049 trial), $\mathrm{N} \mathrm{Meda}^{2}$ (Coordinator of Bobo-Dioulasso Centre), PMsellati ${ }^{1}$ (Coordinator of Abidjan Centre), R Ramon ${ }^{1}$.

Gynaecology-Obstetrics: A Bazié 2 , B Dao 2 , R Likikouet ${ }^{1}$, $\mathrm{L} \mathrm{Mandelbrot}^{4}$ (Principal Investigator), C WelffensEkra $^{1}$ (Principal investigator).

Microbiology: D Bonard ${ }^{1}$, P Combe ${ }^{1}$, M Dosso ${ }^{1}$, L Gautier-Charpentier', FD $\mathrm{Ky}^{2}$, A Ouangré2,

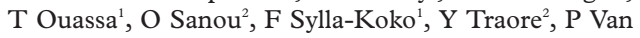
de Perre ${ }^{2}$.

Molecular biology: AM Cassel-Beraud ${ }^{2}$, JB Kottan ${ }^{1}$, O Manigart ${ }^{2}, \mathrm{C}_{\text {Montcho }}{ }^{1}$, C Rouzioux ${ }^{4}$, A Simonon ${ }^{2}$, D Valea ${ }^{2}$, B You ${ }^{1}$.

Paediatrics: R Camara ${ }^{1}$, N Elenga ${ }^{1}$, B Nacro ${ }^{2}$, F Tall', M Timité ${ }^{1}$

Trial monitoring: G Gourvellec ${ }^{1}, \mathrm{O} \mathrm{Ky}-\mathrm{Zerbo}^{2}, \mathrm{~V} \mathrm{Noba}^{1}$, I Sombiéz, S Tiendrebeogo ${ }^{2}$, I Viho', $\mathrm{S}$ Yaro ${ }^{2}$.

Abidjan health facilities: the Anonkoua-Koute, Ouassakara, Yopougon, and Yopougon-Attie health centres, the Centre Hospitalier Universitaire de Yopougon.

Bobo-Dioulasso health facilities: the Accart-Ville, Farakan, and social security health centres, the Centre Hospitalier National Sourô Sanou.

This work was presented in part at the XIth International Conference on AIDS and STDs in Africa, Lusaka, Zambia, 1999 (abstract 13PT31-12).

The authors thank the directors of the health facilities where the study took place and also physicians, nurses, and social the study took place and also physicians, nurses, and social
workers of the DITRAME project for their daily contribution. Workers of the DITRAME project for their daily contribution. The authors wish to acknowledge women participating to this
study; without their collaboration, this research could not have study; without their
been performed.

Sponsorship: Agence Nationale de Recherches sur le SIDA (ANRS), Paris, France.

Contributors: IS, ST, SY, and OK-Z were trial monitors; NM, MC, LM, and FD cowrote the protocol and coordinated the trial; $\mathrm{AO}$ and PvdP contributed to design and conduct of the research and supervised laboratory activities; $\mathrm{BD}$ supervised obstetrical activities; IS wrote the paper, with NM and FD.

1 Gerbase AC, Rowley JT, Mertens TE. Global epidemiology of sexually transmitted diseases. Lancet 1998;351(suppl III) $: 2-4$.

2 Goeman J, Meheus A, Piot P. L'épidémiologie des MST dans les pays en development à l'ère du SIDA. Ann Soc belge Med Trop 1991;71:81-113.

3 Cartoux M, Msellati M, Meda N, et al. Attitude of pregnant women towards HIV testing in Abidjan, Côte d'Ivoire and Bobo-Dioulasso, Burkina Faso. AIDS 1998;12:2337-44.

Young H. Syphilis. Serology. Dermatol Clin 1998;16:691-8

5 Sangaré L, Meda N, Lankoandé S, et al. HIV infection among pregnant women in Burkina Faso: a nationwide serosurvey. Int $\mathcal{F}$ STD AIDS 1997;8:646-51.

6 Blankhart D, Muller O, Gresenguet G, et al. Sexually transmitted diseases in young pregnant women in Bangui, Central African Republic. Int F STD AIDS 1999;10:609-14.

7 Ozumba UC, Oshi DC, Nwokeyi CM, et al. Trends in seroreactivity for syphilis among pregnant Nigerian women. Sex Transm Inf 1999;75:120.

8 Lamptey RP, Kamenga CM, Weir SS. Prevention of sexual transmission of HIV in sub-Saharan Africa: lessons transmission of HIV in sub-Saharan Afr

9 Caraël M, Buvé A, Awusabo-Asare K. The making of HIV epidemics: what are the driving forces? AIDS 1997; 11(supp1 B):S23-31.

10 Fleming DT, Wasserheit JN. From epidemiological synergy to public health policy and practice: the contribution of other sexually transmitted diseases to sexual transmission of HIV infection. Sex Transm Inf 1999;75:3-17.

11 Mayaud P, Mosha F, Todd J, et al. Improved treatment services significantly reduce the prevalence of sexually transmitted diseases in rural Tanzania: results of a randomised controlled trial. AIDS 1997;11:1873-80.

12 Grosskurt H, Mosha F, Todd J, et al. Impact of improved treatment of sexually transmitted diseases on HIV infection in rural Tanzania: randomised controlled trial. Lancet 1995;346:530-6. 